\title{
metabolites
}

ISSN 2218-1989

www.mdpi.com/journal/metabolites/

Article

\section{Reliable Metabolic Flux Estimation in Escherichia coli Central Carbon Metabolism Using Intracellular Free Amino Acids}

\author{
Nobuyuki Okahashi ${ }^{1}$, Shuichi Kajihata ${ }^{1}$, Chikara Furusawa ${ }^{1,2}$ and Hiroshi Shimizu ${ }^{1, *}$ \\ 1 Department of Bioinformatic Engineering, Graduate School of Information Science and \\ Technology, Osaka University, 1-5 Yamadaoka, Suita, Osaka 565-0871, Japan; \\ E-Mails: n-okahashi@ist.osaka-u.ac.jp (N.O.); s-kajihata@ist.osaka-u.ac.jp (S.K.); \\ chikara.furusawa@riken.jp (C.F.) \\ 2 Quantitative Biology Center, RIKEN, 6-2-3 Furuedai, Suita 565-0874, Japan \\ * Author to whom correspondence should be addressed; E-Mail: shimizu@ist.osaka-u.ac.jp; \\ Tel./Fax: +81-6-6879-7446.
}

Received: 17 March 2014; in revised form: 10 May 2014 / Accepted: 20 May 2014 /

Published: 30 May 2014

\begin{abstract}
C}$ metabolic flux analysis (MFA) is a tool of metabolic engineering for investigation of in vivo flux distribution. A direct ${ }^{13} \mathrm{C}$ enrichment analysis of intracellular free amino acids (FAAs) is expected to reduce time for labeling experiments of the MFA. Measurable FAAs should, however, vary among the MFA experiments since the pool sizes of intracellular free metabolites depend on cellular metabolic conditions. In this study, minimal ${ }^{13} \mathrm{C}$ enrichment data of FAAs was investigated to perform the FAAs-based MFA. An examination of a continuous culture of Escherichia coli using ${ }^{13} \mathrm{C}$-labeled glucose showed that the time required to reach an isotopically steady state for FAAs is rather faster than that for conventional method using proteinogenic amino acids (PAAs). Considering $95 \%$ confidence intervals, it was found that the metabolic flux distribution estimated using FAAs has a similar reliability to that of the PAAs-based method. The comparative analysis identified glutamate, aspartate, alanine and phenylalanine as the common amino acids observed in E. coli under different culture conditions. The results of MFA also demonstrated that the ${ }^{13} \mathrm{C}$ enrichment data of the four amino acids is required for a reliable analysis of the flux distribution.
\end{abstract}

Keywords: metabolic flux analysis; intracellular free amino acids; Escherichia coli; GC-MS 


\section{Introduction}

Metabolic flux analysis (MFA) is a tool driving metabolic engineering through a detailed understanding of intracellular carbon flux distributions in various organisms [1-5]. The level of flux in each reaction is estimated by a tracer labeling experiment using, such as ${ }^{13} \mathrm{C}$-labeled glucose, as the labeling patterns of the intracellular metabolites reflect the flux distribution inside the cells [6-9]. In conventional MFA, the flux distribution is estimated from the ${ }^{13} \mathrm{C}$ enrichment of proteinogenic amino acids (PAAs) determined by gas chromatography-mass spectrometry (GC-MS) or nuclear magnetic resonance (NMR) [10,11]. Whereas a large amount of PAAs in the cells is preferable for a precise determination of the ${ }^{13} \mathrm{C}$ enrichment, the slow turnover of PAAs has restricted MFA applications, since a relatively long experimental time, for example, 5 residence time in continuous culture, is required for the complete labeling of the PAAs.

For the MFA study of batch and fed-batch culture, intracellular free amino acids (FAAs) with faster turnover rates are more promising targets for a ${ }^{13} \mathrm{C}$ enrichment measurement [12-16]. The time course analysis of ${ }^{13} \mathrm{C}$ enrichment of FAAs demonstrated that the experimental time required to reach an isotopic steady state of the FAAs is 2 to 10 times faster than that for PAAs [17]. However, the smaller pool sizes and large compositional variations of FAAs are expected to affect the design of an MFA experiment [18]. This is because a confidence interval of flux estimation depends on a set of measureable FAAs. The relationship between the set of measurable data and resulting confidence intervals are not well investigated from the aspect of experimental data. The large sampling volume of broth required for broader amino acid coverage would disturb a constant culture condition. Understanding the required dataset for reliable MFA will be a useful guideline for the design of MFA experiments using FAAs.

In this study, minimal ${ }^{13} \mathrm{C}$ enrichment data of FAAs to perform the FAAs-based MFA was investigated by the analysis of Escherichia coli. It was realized that four amino acids, i.e., glutamate (Glu), aspartate (Asp), alanine (Ala), and phenylalanine (Phe), are the most commonly observed in E. coli at distinct culture conditions. The results of MFA also demonstrated that the ${ }^{13} \mathrm{C}$ enrichment data of the four amino acids is required for a reliable analysis of the flux distribution.

\section{Results and Discussion}

\subsection{Continuous Culture of E. coli MG1655}

In this study, FAAs-based MFA was developed by using E. coli MG1655 strain. E. coli is one of the most widely-used model organisms in the fields of metabolic engineering and systems biology. In order to compare the PAAs- and FAAs-based methods, ${ }^{13} \mathrm{C}$ enrichment of PAAs and FAAs were determined for $E$. coli cells obtained from a same culture. The continuous culture of $E$. coli cells was performed at a dilution rate of $0.2 \mathrm{~h}^{-1}$ using a synthetic medium containing glucose. After 8 residence time, the metabolic steady state was confirmed from constant levels of $\mathrm{OD}_{600}$, concentration of $\mathrm{O}_{2}$ and $\mathrm{CO}_{2}$ in exhausted gas and concentration of organic acids in medium (Figure S1). Specific uptake rate of glucose and the specific production rate of acetate, formate, lactate, and ethanol in the metabolic steady state were $3.41 \pm 0.01,1.13 \pm 0.06,0.98 \pm 0.05,0.09 \pm 0.01$, and $0.01 \pm 0.01 \mathrm{mmol} / \mathrm{g}-\mathrm{DCW} / \mathrm{h}$, respectively. After attaining the metabolic steady state, the carbon source in the feeding medium was 
changed from $100 \%$ natural glucose to a mixture of glucose containing $1.0 \%$ non-labeled glucose, $49.2 \%\left[1-{ }^{13} \mathrm{C}\right]$ glucose and $49.8 \%\left[\mathrm{U}-{ }^{13} \mathrm{C}\right]$ glucose. The $E$. coli cells were repeatedly collected from the culture, and the ${ }^{13} \mathrm{C}$ enrichment of PAAs and FAAs were determined using the GC-MS analysis. Time course of ${ }^{13} \mathrm{C}$ enrichment of representative PAAs and FAAs are shown in Figure 1. While an isotopic steady state was attained at $25 \mathrm{~h}$ ( 5 residence time) after the start of ${ }^{13} \mathrm{C}$ labeling for the case of PAAs, the ${ }^{13} \mathrm{C}$ enrichment of FAAs reached plateau in $10 \mathrm{~h}$ ( 2 residence time). This result confirms that the FAAs-based method can reduce labeling time for MFA. Although the faster turnover rate for FAAs is preferable for an MFA study of batch and fed-batch culture [12-15], it remains unclear whether a reliable result can be produced by FAAs-based MFA. It is not also obvious which FAAs are reproducibly observed from $E$. coli cells in various culture conditions, and whether a precise metabolic flux can be estimated by using the observed amino acids.

Figure 1. Time course of ${ }^{13} \mathrm{C}$ enrichment of proteinogenic and free amino acids. Fractional labeling of proteinogenic amino acids (PAAs, Closed circle) and free amino acids (FAAs, Closed square) are represented. Fractional labeling is a ratio of labeled carbons in the metabolite. Other data are shown in Figure S2.
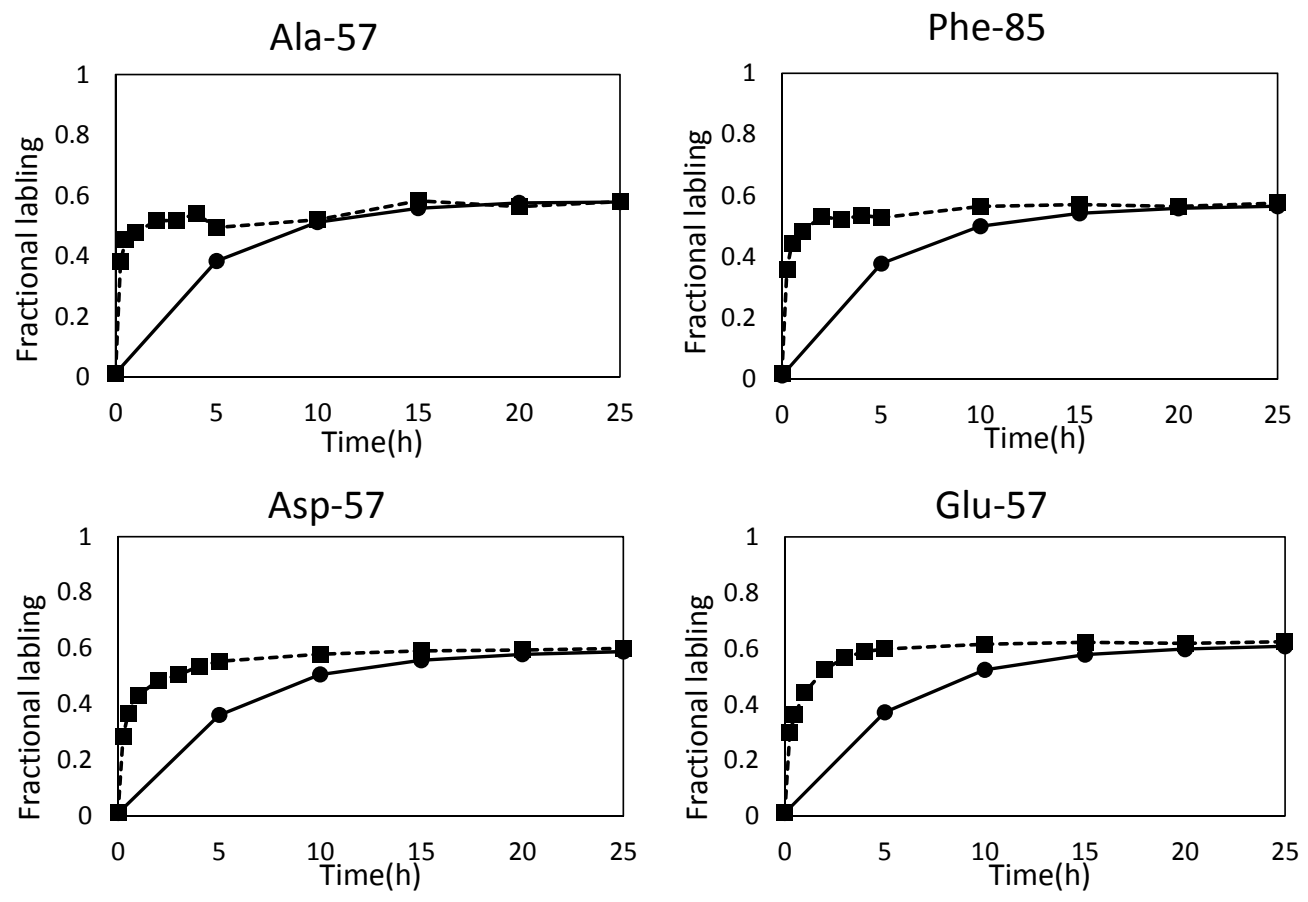

\subsection{Metabolic Flux Analysis}

Performance of FAAs-based MFA method was investigated by comparison with that of PAAs-based method. For this purpose, a reference metabolic flux distribution was determined by the PAAs-based MFA. From the E. coli cells obtained at $25 \mathrm{~h}$ after the start of the tracer experiment, PAAs were prepared via acid hydrolysis of the proteins. The GC-MS analysis of the derivatized samples successfully determined the ${ }^{13} \mathrm{C}$ enrichment of 25 fragments derived from a total of 11 amino acids (Table 1). Using all of the ${ }^{13} \mathrm{C}$ enrichment data (PAAs_fullset), a reference metabolic flux distribution was estimated by a non-linear fitting to a metabolic model (Tables S1-1 and S1-2). The estimated and measured ${ }^{13} \mathrm{C}$ enrichment were almost similar to each other, indicating that a reasonable 
flux distribution was estimated from the best-fitted results (Figure 2a). Figure 3 shows the estimated fluxes in their representative reactions. The results of PAAs_fullset ( $\mathrm{a}$ in Figure 3) show that 75\% of glucose is catabolized via the glycolysis (PGI net flux) and $25 \%$ via the pentose phosphate (PP) pathway (G6PDH flux). The Entner-Doudoroff (ED) pathway is inactive in this condition. While a significant amount of pyruvate (Pyr) and acetyl-CoA (AcCoA) are secreted to the medium as formate and acetate, the remaining carbon flows into the TCA cycle. The flux distribution is essentially comparable to a previously reported result [17]. In this study, 95\% confidence intervals of each flux were estimated by the grid search method (represented as error bars in Figure 3 ). The $95 \%$ confidence intervals of glycolysis/PP pathway branch point were deduced to be \pm 3 , and the flux intervals of upper glycolysis, PP pathway and ED pathway were estimated to be within \pm 6 . Relatively large $95 \%$ confidence intervals $( \pm 13)$ were observed for fluxes in lower glycolysis, TCA cycle, glyoxylate shunt and anaplerosis.

Table 1. Data points used for metabolic flux analysis and residual sum of squares (RSS) of best-fitted results.

\begin{tabular}{lcccccc}
\hline & $\begin{array}{c}\text { Number of } \\
\text { independent } \\
\text { measurements } \\
(=\boldsymbol{n})\end{array}$ & $\begin{array}{c}\text { Number of } \\
\text { metabolites } \\
\text { used for } \\
\text { fitting }\end{array}$ & $\begin{array}{c}\text { Number of } \\
\text { fragments } \\
\text { used for } \\
\text { fitting }\end{array}$ & $\boldsymbol{n - p} \boldsymbol{p}^{\mathbf{1}}$ & RSS & RSS/(n-p) \\
\hline PAAs_fullset & 92 & 11 & 25 & 71 & 0.0013 & 0.00002 \\
FAAs_fullset & 66 & 9 & 19 & 45 & 0.0037 & 0.00008 \\
FAAs_Glu+Asp & 25 & 2 & 7 & 9 & 0.0007 & 0.00017 \\
FAAs_Glu+Asp+Ala & 30 & 3 & 9 & 19 & 0.0012 & 0.00014 \\
FAAs_Glu+Asp+Ala+Phe & 40 & 4 & 11 & 4 & 0.0015 & 0.00008 \\
\hline
\end{tabular}

Figure 2. Comparison of measured and estimated ${ }^{13} \mathrm{C}$ enrichment of amino acids. The $\mathrm{X}$ axis and $\mathrm{Y}$ axis indicate measured and estimated ${ }^{13} \mathrm{C}$ enrichment, respectively, of the (a) PAAs_fullset; (b) FAAs_fullset; (c) FAAs_Glu+Asp; (d) FAAs_Glu+Asp+Ala; and (e) FAAs_Glu+Asp+Ala+Phe.
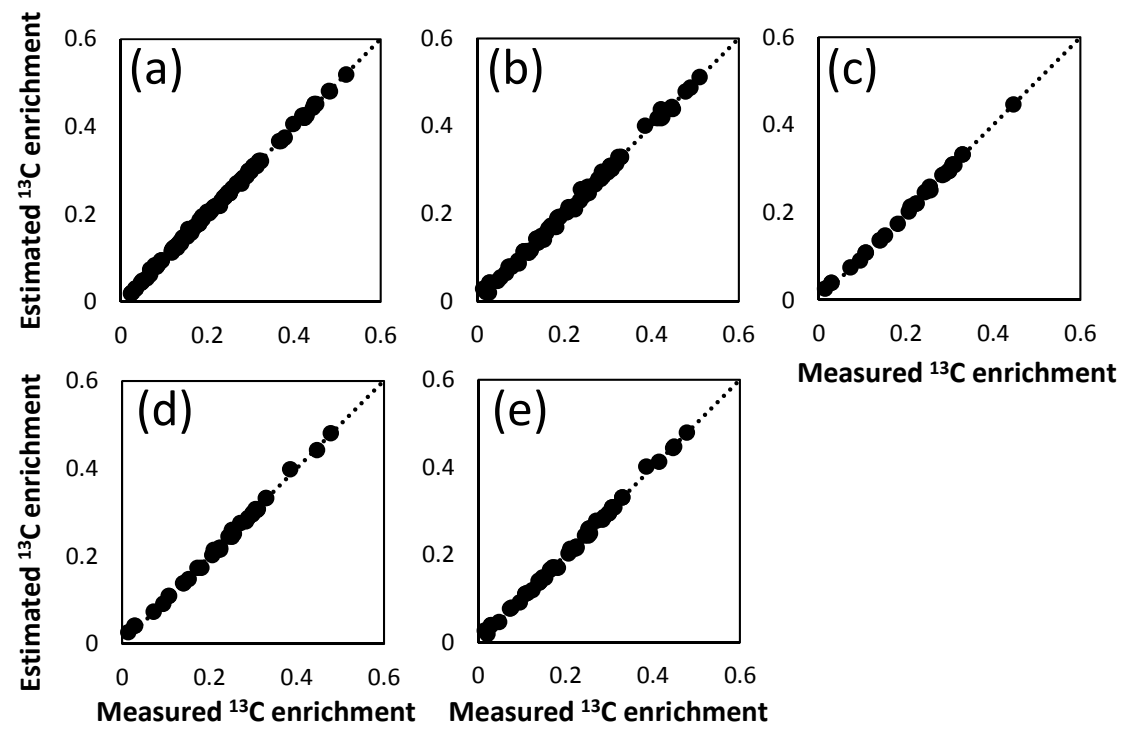
Figure 3. Comparison of metabolic flux distributions estimated using different datasets. Flux values are normalized to a glucose uptake rate of 100 . Fluxes and $95 \%$ confidence intervals in representative reactions are shown. Full data is available from Tables S1-1, S2-1, S3-1, S4-1 and S5-1.

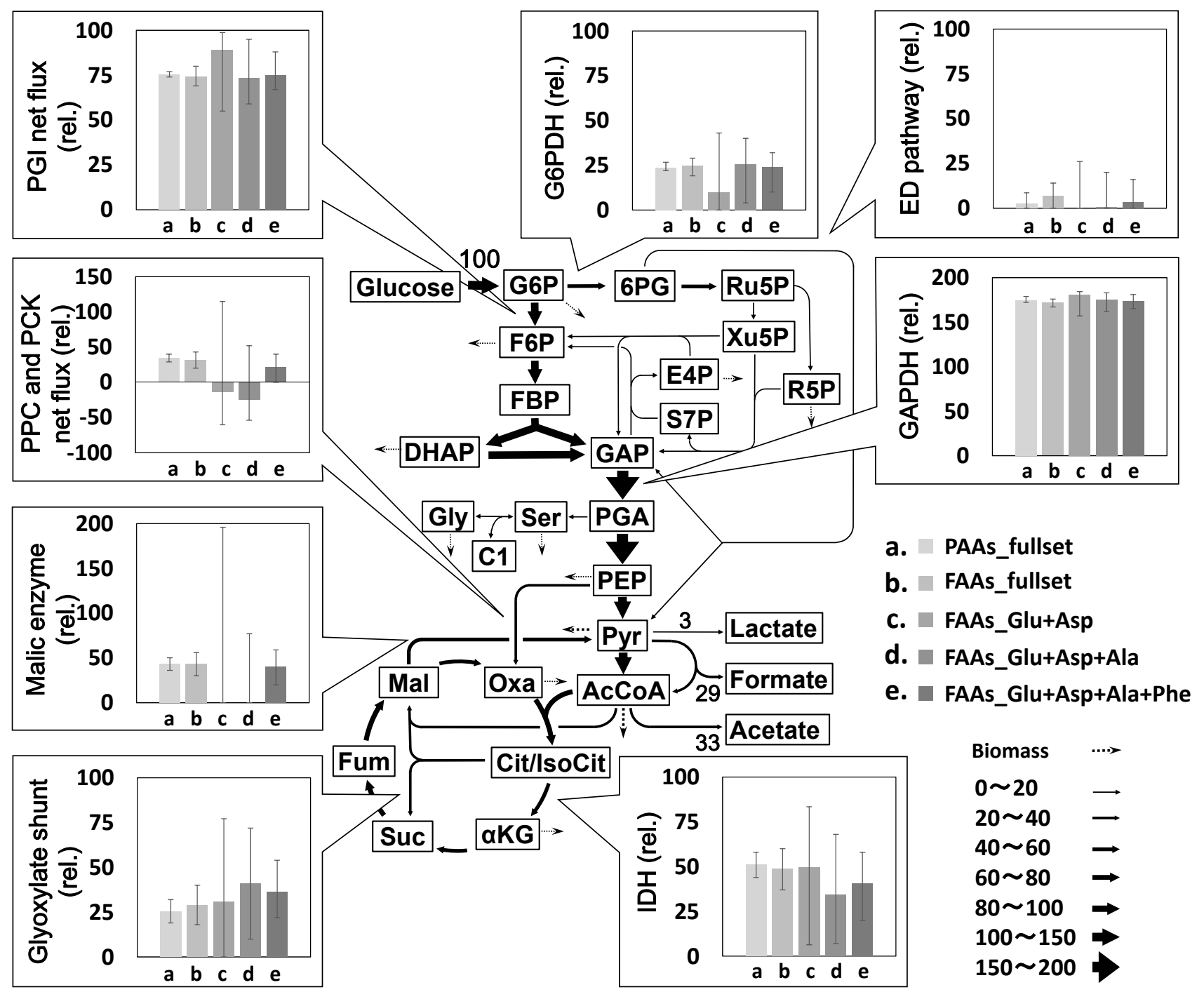

The arrow width represents the flux levels estimated by using the reference (PAAs_fullset) dataset. Abbreviations: PGI: phosphoglucose isomerase, PPC: phosphoenolpyruvate carboxylase, PCK: phosphoenolpyruvate carboxykinase, G6PDH: glucose 6-phosphate dehydrogenase, GAPDH: glyceraldehyde 3-phosphate dehydrogenase, IDH: isocitrate dehydrogenase, G6P: glucose 6-phosphate, F6P: fructose 6-phosphate, FBP: fructose 1,6-bisphosphate, DHAP: dihydroxyacetone phosphate, GAP: glyceraldehyde 3-phosphate, PGA: 3-phosphoglycerate, PEP: phosphoenolpyruvate, Pyr: pyruvate, AcCoA: acetyl-coenzyme A, Cit: citrate, $\alpha \mathrm{KG}: \alpha$-ketoglutarate, Suc: succinate, Fum: fumarate, Mal: malate, Oxa: oxaloacetate, 6PG: 6-phosphogluconate, Ru5P: ribulose 5-phosphate, R5P: ribose 5-phosphate, Xu5P: xylulose 5-phosphate, S7P: sedoheptulose 7-phosphate, E4P: erythrose 4-phosphate, Ala: alanine, Phe: phenylalanine, Asp: aspartate, Glu: glutamate. 
From the same E. coli culture, a flux distribution was estimated using FAAs. Intracellular FAAs were extracted from the E. coli cells at 15, 20, and $25 \mathrm{~h}$ after the start of the tracer labeling. The ${ }^{13} \mathrm{C}$ enrichment of 19 fragments derived from 11 amino acids was determined with signal to noise ratio $>10$ by GC-MS analysis (FAAs_fullset). A flux distribution was successfully estimated by a non-linear fitting of the metabolic model to the mean ${ }^{13} \mathrm{C}$ enrichment data (Figure 2b, Tables S2-1 and S2-2). As shown in Figure 3, the metabolic flux distribution estimated from FAAs data (FAAs_fullset, $\mathrm{b}$ in Figure 3) was quite similar to the reference data determined by PAAs-based MFA (PAAs_fullset, a in Figure 3).

An investigation of the continuous culture of $E$. coli using a simplified metabolic model demonstrated that PAAs- and FAAs-based MFA methods can produce compatible flux distributions [17]. The MFA performed in this study using the generally accepted metabolic model confirmed that essentially identical flux distributions are estimated from the ${ }^{13} \mathrm{C}$ enrichment data of PAAs (PAAs_fullset) and FAAs (FAAs_fullset), with the 95\% confidence intervals of the two sets overlapping each other (Figures 2 and 3).

A comparison of the confidence intervals also reveals that a flux distribution estimated from the FAAs_fullset shows wider confidence intervals (Figure 3). It is because the number of measurable fragments, amino acids and independent measurements of the FAAs_fullset $(n=66)$ is significantly less than that for the PAAs_fullset $(n=92$, Table 1$)$. The smaller amount of ${ }^{13} \mathrm{C}$ enrichment data cannot be overlooked in the FAAs-based method when directly analyzing FAAs with low concentrations.

\subsection{Combination of Amino Acids for Reliable FAAs-Based MFA}

Measurable FAAs should vary among the FAA-based MFA experiments since the pool sizes of intracellular free metabolites depend on cellular metabolic conditions. For instance, it was reported that the pool size of FAAs in E. coli ranged from $2 \times 10^{-2}$ to $80 \mu \mathrm{mol} / \mathrm{g}-\mathrm{DCW}$ [18]. Indeed, previous studies demonstrated that distinct sets of FAAs were observed in the FAAs-based MFA at different culture conditions (Table 2) [14,17,19]. For the amino acids shown in Table 2, Glu, Asp, Ala, and Phe are the four amino acids commonly observed in the four studies, suggesting that these amino acids are likely to be measurable from E. coli cells at various culture conditions (Table 2). These amino acids were also commonly observed in various single gene knock-out E. coli strains [20]. In the GC-MS data obtained in this study, the signal of glutamate is rather intense than for the other amino acids (Figure 4). The second, third and fourth most intense signals are from Asp, Ala, and Phe, respectively. A similar tendency was also reported [20], indicating that glutamate is a useful amino acid to investigate for the ${ }^{13} \mathrm{C}$ enrichment of $\alpha$-ketoglutarate $(\alpha \mathrm{KG})$ in FAAs-based MFA.

In order to test the performance of FAA-based MFA experiment using Glu, Asp, Ala, and Phe, flux distributions were estimated using the ${ }^{13} \mathrm{C}$ enrichment data of 7 fragments of glutamate and aspartate (FAAs_Glu+Asp), 9 fragments of glutamate, aspartate, and alanine (FAAs_Glu+Asp+Ala), and 11 fragments of glutamate, aspartate, alanine and phenylalanine (FAAs_Glu+Asp+Ala+Phe), respectively (Table 1). The results obtained from each dataset are shown in Figures 2c-e and 3, Tables S3-1, S3-2, S4-1, S4-2, S5-1 and S5-2. The comparison of the results with the reference data (PAAs_fullset) revealed that, in the case of the FAAs_Glu+Asp (c in Figure 3) and FAAs_Glu+Asp+Ala (d in Figure 3) datasets, very large $95 \%$ confidence intervals are observed especially for flux of the malic enzyme 
reaction and phosphoenolpyruvate carboxylase (PPC) and phosphoenolpyruvate carboxykinase (PCK) net flux (Figure 3). On the other hand, the FAAs_Glu+Asp+Ala+Phe (e in Figure 3) dataset produced a rather precise metabolic flux distribution with narrower confidence intervals. For example, the $95 \%$ confidence interval of PPC and PCK net flux and malic enzyme flux determined by FAAs_Glu+Asp+Ala+Phe were $38 \%$ and $51 \%$ of that of FAAs_Glu+Asp+Ala, respectively. This result indicates that Glu, Asp, Ala, and Phe constitute a practical minimal set for FAAs-based MFA.

Table 2. Free amino acids measured in ${ }^{13} \mathrm{C}$-metabolic flux analysis studies using E. coli.

\begin{tabular}{|c|c|c|c|c|}
\hline & This study & $\begin{array}{l}\text { Mori et al. } \\
\text { (2011) [17] }\end{array}$ & $\begin{array}{l}\text { Toya et al. } \\
\text { (2010) [19] }\end{array}$ & $\begin{array}{c}\text { Iwatani et al. } \\
(2007)[14]\end{array}$ \\
\hline \multicolumn{5}{|c|}{ Experimental conditions } \\
\hline Analysis & GC-MS & GC-MS & CE-TOFMS & LC-MS/MS \\
\hline Culture & $\begin{array}{c}\text { Chemostat } \\
\text { culture }\end{array}$ & $\begin{array}{c}\text { Chemostat } \\
\text { culture }\end{array}$ & $\begin{array}{l}\text { Batch } \\
\text { culture }\end{array}$ & $\begin{array}{l}\text { Fed-batch } \\
\text { culture }\end{array}$ \\
\hline \multicolumn{5}{|c|}{ Amino acids } \\
\hline Alanine & + & + & + & + \\
\hline Valine & + & - & + & + \\
\hline Leucine & + & + & + & - \\
\hline Isoleucine & - & - & + & - \\
\hline Lysine & - & - & + & - \\
\hline Aspartate & + & + & + & + \\
\hline Asparagine & - & - & - & + \\
\hline Threonine & + & - & + & + \\
\hline Methionine & - & - & - & - \\
\hline Glutamate & + & + & + & + \\
\hline Glutamine & - & - & - & + \\
\hline Arginine & - & - & + & - \\
\hline Proline & - & - & + & - \\
\hline Glycine & + & - & + & + \\
\hline Serine & - & + & + & + \\
\hline Cysteine & - & - & - & - \\
\hline Histidine & - & - & + & - \\
\hline Tyrosine & + & - & + & + \\
\hline Phenylalanine & + & + & + & + \\
\hline Tryptophan & - & - & - & - \\
\hline
\end{tabular}

+: detected; -: not detected.

According to the study of flux ratio analysis reported by Fischer and Sauer [21], labeling patterns of ${ }^{13} \mathrm{C}$ enrichment of Pyr and oxaloacetate (Oxa) are essential to elucidate the branch ratio of glycolysis, PP pathway and ED pathway or TCA cycle and glyoxylate shunt. This indicates that ${ }^{13} \mathrm{C}$-labeling of Ala and Asp synthesized from Pyr and Oxa, respectively, is necessary to estimate flux in various flux distributions in the central carbon metabolism. The metabolic flux analysis using FAAs_Glu+Asp and FAAs_Glu+Asp+Ala shows that, whereas levels of PGI and GAPDH net fluxes are similar to that of the reference data (PAA_fullset), large confidence intervals are observed than for the case of the other metabolic fluxes (Figure 3). The result indicates that, in addition to the ${ }^{13} \mathrm{C}$ enrichment information of 
$\alpha \mathrm{KG}$, Oxa, and Pyr derived from Glu, Asp, and Ala, respectively, an analysis of other amino acids is required for a more precise estimation of the metabolic flux distribution. The confidence intervals of metabolic flux analysis were drastically improved by employing the FAAs_Glu+Asp+Ala+Phe dataset, indicating a requirement for ${ }^{13} \mathrm{C}$ enrichment information of PEP and E4P in Phe (Figure 3). Indeed, it has been reported that levels of metabolic flux in anaplerotic pathways can be well estimated from PEP in addition to Pyr, Oxa, and $\alpha \mathrm{KG}$ [21].

Figure 4. Signal intensities of free amino acids determined by GC-MS. Total signal intensities of Glu ([M-57], [M-85], [M-159]), Asp ([M-57], [M-85], [M-159], [f302]), Ala ([Ala-57], [Ala-85]) and Phe ([M-85], [f302]) are shown in the figure.

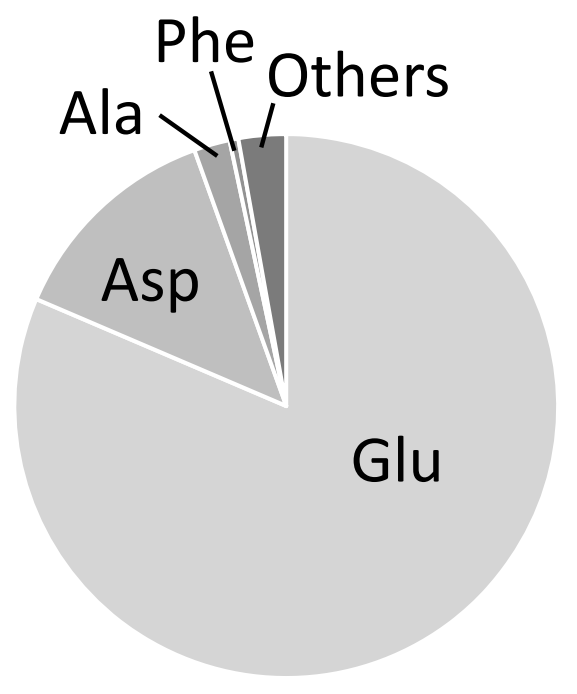

These results suggest that a combination of Ala, Asp, Glu, and Phe synthesized from Pyr, Oxa, $\alpha \mathrm{KG}, \mathrm{PEP}$, and E4P is a requirement for FAAs-based MFA using $\left[1-{ }^{13} \mathrm{C}\right]$ and $\left[\mathrm{U}_{-}{ }^{13} \mathrm{C}\right]$ glucose. This mixture of ${ }^{13} \mathrm{C}$-labeled glucose was generally used in various MFA studies [1,12-14,17,19,21,22]. Since there are significant fluxes in each reaction in the metabolic network shown in Figure 3, the four amino acids should contain enough information to determine metabolic fluxes in other culture conditions. The additional analysis of other amino acids (Table 2) is expected to improve the confidence intervals of the estimated metabolic fluxes. The findings in this study are most applicable for GC-MS analysis and further experiments would be needed to show that this knowledge is also applicable to MFA based on intermediate metabolites using LC-MS. Although additional confirmations are required, our approach also would be available to other microorganisms with similar metabolic pathway, such as Bacillus subtilis and Corynebacterium glutamicum.

\section{Experimental Section}

\subsection{Strain and Medium}

Escherichia coli K-12 MG1655 strain was cultured in M9 medium consisting of $17.1 \mathrm{~g} / \mathrm{L}$ $\mathrm{Na}_{2} \mathrm{HPO}_{4} \bullet 12 \mathrm{H}_{2} \mathrm{O}, 3 \mathrm{~g} / \mathrm{L} \mathrm{KH}_{2} \mathrm{PO}_{4}, 0.5 \mathrm{~g} / \mathrm{L} \mathrm{NaCl}, 2 \mathrm{~g} / \mathrm{L} \mathrm{NH} 4 \mathrm{Cl}, 123 \mathrm{mg} / \mathrm{L} \mathrm{MgSO}{ }_{4} \cdot 7 \mathrm{H}_{2} \mathrm{O}, 2.78 \mathrm{mg} / \mathrm{L}$ $\mathrm{FeSO}_{4} \cdot 7 \mathrm{H}_{2} \mathrm{O}, 14.7 \mathrm{mg} / \mathrm{L} \mathrm{CaCl} \mathrm{Ca}_{2} \cdot 2 \mathrm{H}_{2} \mathrm{O}, 10 \mathrm{mg} / \mathrm{L}$ thiamine-hydrochloride and $5 \mathrm{~g} / \mathrm{L}$ glucose. 
Adecanol $\left(1 \times 10^{-3} \% w / w\right)$ was added as an antiform in the continuous culture. $\left[1-{ }^{13} \mathrm{C}\right]$ glucose $(98 \%-99 \%)$ and $\left[\mathrm{U}_{-}{ }^{13} \mathrm{C}\right]$ glucose $(99 \%)$ were purchased from Cambridge Isotope Laboratories (Andover, MA, USA).

\subsection{Culture Condition}

A frozen stock of $E$. coli cells was inoculated in $40 \mathrm{~mL}$ M9 medium and incubated for $14 \mathrm{~h}$ at $37^{\circ} \mathrm{C}$ with reciprocal shaking. Continuous culture was performed in a $1 \mathrm{~L}$ bioreactor (ABLE, Tokyo, Japan) equipped with temperature, $\mathrm{pH}$, dissolved oxygen, and exhaust gas $\left(\mathrm{O}_{2}\right.$ and $\left.\mathrm{CO}_{2}\right)$ sensors. The working volume and aeration rate were $400 \mathrm{~mL}$ and $400 \mathrm{~mL} / \mathrm{min}$, respectively. The temperature was controlled at $37{ }^{\circ} \mathrm{C}$, and $\mathrm{pH}$ was set at 7.0 using an $\mathrm{NH}_{3}$ solution. Five hours after inoculation, the continuous culture was started at a dilution rate of $0.2 \mathrm{~h}^{-1}$. The feeding medium containing natural glucose was replaced with one containing ${ }^{13} \mathrm{C}$-labeled glucose after the continuous culture reached a metabolic steady state. Labeling of glucose in the medium was determined by gas chromatography-mass spectrometry (GC-MS) analysis by a previously described method [22].

\subsection{Off-Line Measurements}

Levels of $\mathrm{OD}_{600}$ were determined using a spectrophotometer (UVmini-1240, Shimadzu, Kyoto, Japan). A glucose sensor (Bioanalyzer BF-5, Oji Scientific Instruments, Hyogo, Japan) was used for glucose analysis. Ethanol concentration was measured using a gas chromatograph (Agilent 7890A GC; Agilent Technologies, Santa Clara, USA) operated at the following conditions; column, Stabilwax $60 \mathrm{~m} \times 0.32 \mathrm{~mm}$ ID $\times 1 \mu \mathrm{m}$ (Restek, Bellefonte, USA); carrier gas, helium; flow rate, $6.5 \mathrm{~mL} / \mathrm{min}$; injection volume, $1 \mu \mathrm{L}$; split ratio, $1: 10$; oven temperature, $70{ }^{\circ} \mathrm{C}$ for $3 \mathrm{~min}$ and raised at $10{ }^{\circ} \mathrm{C} / \mathrm{min}$; FID detector temperature, $250^{\circ} \mathrm{C}$. The concentrations of organic acids (lactate, formate, acetate, succinate and fumarate) were determined by a high-performance liquid chromatography (HPLC) system (Prominence; SHIMADZU, Kyoto, Japan, column, Oapak-A; $7.8 \mathrm{~mm}$ ID × $30 \mathrm{~cm}$ (TOSOH, Tokyo, Japan); column temperature, $40{ }^{\circ} \mathrm{C}$; mobile phase, $0.75 \mathrm{mM} \mathrm{H}_{2} \mathrm{SO}_{4}$ in water; flow rate, $0.8 \mathrm{~mL} / \mathrm{min}$; detection, UV $210 \mathrm{~nm}$ ).

\subsection{Sample Preparation for GC-MS Analysis}

For analysis of the PAAs, $10 \mathrm{~mL}$ broth culture was taken directly from the reactor and centrifuged at $9800 \times \mathrm{g}$ for $10 \mathrm{~min}$ at $4{ }^{\circ} \mathrm{C}$. The cell pellet was washed twice with $0.9 \% \mathrm{NaCl}$ and hydrolyzed in $2 \mathrm{~mL} 6 \mathrm{~N} \mathrm{HCl}$ at $105{ }^{\circ} \mathrm{C}$ for $18 \mathrm{~h}$. After filtration (Cosmonice filter W, pore size $0.45 \mu \mathrm{m}$, Filter diameter $13 \mathrm{~mm}$, Nacalai Tesque, Kyoto, Japan), $10 \mu \mathrm{L}$ of internal standard (600 $\mu \mathrm{M}$ cycloleucine) was added to $10 \mu \mathrm{L}$ of the hydrolysate and evaporated to dryness. The dried residue was dissolved in $50 \mu \mathrm{L}$ acetonitrile and $50 \mu \mathrm{L} \mathrm{N}$-(tert-butyldimethylsilyl)- $N$-methyl-trifluoroacetamide containing $1 \%$ tert-butyldimethylchlorosilane, and incubated at $105{ }^{\circ} \mathrm{C}$ for $1 \mathrm{~h}$. After $1 \mathrm{~h}$ cooling, a supernatant was injected for GC-MS analysis. Intercellular FAAs were extracted by the following procedure. Cells were collected by filtration of $5 \mathrm{~mL}$ of culture broth (PTFE type membrane, $0.5 \mu \mathrm{m}$ pore size, diameter $90 \mathrm{~mm}$, ADVANTEC, Tokyo,Japan) [23,24]. Cells on the filter were immediately immersed in $1.6 \mathrm{~mL}$ methanol $\left(-80^{\circ} \mathrm{C}\right)$ and preserved at $-80^{\circ} \mathrm{C}$. After the frozen cell sample was suspended in $1.6 \mathrm{~mL}$ of chloroform $\left(-30^{\circ} \mathrm{C}\right), 630 \mu \mathrm{L}$ Milli-Q water $\left(4^{\circ} \mathrm{C}\right)$ and $10 \mu \mathrm{L} 600 \mu \mathrm{M}$ cycloleucine dissolved in water 
were added. After vortexing and sonication for $1 \mathrm{~min}$, the mixture was centrifuged at $3700 \times \mathrm{g}$ for 40 min at $4{ }^{\circ} \mathrm{C}$. A $2 \mathrm{~mL}$ aqueous layer was evaporated to dryness (Speed Vac, Thermo Schientific, Waltham, Japan).

\subsection{GC-MS Analysis of PAAs and FAAs}

The mass isotopic distributions of four type of ion clusters at mass to charge $(\mathrm{m} / \mathrm{z})$ rations of [M-57], [M-85], [M-159], and [f302] derived from each amino acid were determined by using GC-MS (Agilent 7890A GC and 5975C Mass Selective Detector (Agilent Technologies, Santa Clara, USA); column, DB-5MS+DG; $30 \mathrm{~m} \times 0.25 \mathrm{~mm}$ ID $\times 0.25 \mu \mathrm{m}$; (Agilent Technologies, Santa Clara, USA); carrier gas, Helium; flow rate, $1.0 \mathrm{~mL} / \mathrm{min}$; detection mode, selected ion monitoring; ion source temperature, $230{ }^{\circ} \mathrm{C}$; electron impact ionization, $70 \mathrm{eV}$ ). PAAs were analyzed under the following conditions: injection volume, $1 \mu \mathrm{L}$; split ratio, 1:10; oven temperature, $150{ }^{\circ} \mathrm{C}$ for $2 \mathrm{~min}$, increased by $3{ }^{\circ} \mathrm{C} / \mathrm{min}$ to $270{ }^{\circ} \mathrm{C}$, then increased at a rate of $10^{\circ} \mathrm{C} / \mathrm{min}$ to $300{ }^{\circ} \mathrm{C}$, and maintained at that temperature for 5 min [17]. For the analysis of FAAs, splitless mode was employed to measure small amount of FAAs. Detailed conditions are as follows: injection volume, $1 \mu \mathrm{L}$; splitless mode; oven temperature, $60{ }^{\circ} \mathrm{C}$ for $2 \mathrm{~min}$, increased by $20{ }^{\circ} \mathrm{C} / \mathrm{min}$ to $150{ }^{\circ} \mathrm{C}$, then increased at a rate of $3{ }^{\circ} \mathrm{C} / \mathrm{min}$ to $270{ }^{\circ} \mathrm{C}$, further increased to $300{ }^{\circ} \mathrm{C}$ at $10{ }^{\circ} \mathrm{C} / \mathrm{min}$ and maintained at that temperature for $5 \mathrm{~min}$.

\subsection{Metabolic Flux Analysis}

A metabolic model including glycolysis, the tricarboxylic acid (TCA) cycle, glyoxylate shunt, anaplerosis, the pentose phosphate (PP) pathway, the Entner-Doudoroff (ED) pathway, $\mathrm{CO}_{2}$ exchange and $\mathrm{C} 1$ metabolism was employed for flux analysis [21]. The number of free flux or degree of freedom of the model was 21 (Table S1). The fluxes for biomass synthesis of E. coli were calculated from the precursor requirement [25]. The effect of naturally occurring isotopes was removed from the raw mass spectrometry data to obtain corrected ${ }^{13} \mathrm{C}$ labeling patterns of the carbons in the amino acids [26]. The fragments of amino acids were chosen according to the report of Antoniewicz et al. [27]. Additionally, the appropriate fragments were carefully screened by comparing the natural and theoretical isotope abundance ratios. The calculation of fluxes was performed using the in-house software OpenMebius [28], which is a flux estimation tool using the elementary metabolite units (EMU) framework in Matlab 2011b [29]. In short, the fluxes were estimated by minimizing the residual sum of squares (RSS) between the experimentally measured ${ }^{13} \mathrm{C}$ enrichment and the estimated value using the fmincon optimization solver in the Matlab toolbox. The optimizing function is described as:

$$
R S S=\sum_{i=1}^{N}\left(M_{i}^{\text {measured }}-\text { MID }_{i}^{\text {estimated }}\right)
$$

where $M I D_{i}^{\text {measured }}$ is the mass isotopomer distribution (MID) of the $i$ th measured metabolite, $M I D_{i}^{\text {estimated }}$ is the estimated MID of the corresponding metabolite, and $N$ is the number of metabolites used for flux estimation. Optimization was started from 20 sets of random flux distributions. Confidence intervals were calculated by a grid search method as described previously [30-32]. The metabolic flux of reaction $r$ is fixed to $v_{o p t, r}+d$ and the objective function is re-optimized. Here, $v_{o p t, r}$ is the optimized metabolic flux of reaction $r$ and $d$ is the perturbation level. The procedure is iterated 
with increased or decreased $d$. The range of fixed metabolic flux whose RSS is less than the threshold level is the confidence interval. The threshold level is determined by:

$$
R S S_{\text {min_fixed }} \leq R S S_{\text {min }}+\frac{R S S_{\text {min }}}{n-p} F_{\alpha}(1, n-p)
$$

where $R S S_{\text {min fixed }}$ is the minimized RSS with one fixed flux, $R S S_{\text {min }}$ is the original minimized RSS, $n$ is the number of independent data points used in the fitting, $p$ is the degrees of freedom in the original flux fit, $F$ is the $F$-distribution, and $\alpha$ is the confidence level.

\section{Conclusions}

In this study, a performance of the metabolic flux analysis using free amino acids (FAAs) was directly compared with that using proteinogenic amino acids (PAAs). An estimation of $95 \%$ confidence intervals for the first time demonstrated that the FAAs-based MFA has essentially identical reliability with that obtained from the PAAs-based method (Figure 3). It was also experimentally demonstrated that the amino acids derived from Pyr, Oxa, $\alpha \mathrm{KG}, \mathrm{PEP}$, and E4P are at least required for FAAs-based MFA. The minimal set of amino acids for precise MFA can be a practical guide for the design of MFA experiments using FAAs.

\section{Acknowledgments}

We thank Yoshihiro Toya and Fumio Matsuda for helpful comments. This research was partially supported by JST, Strategic International Collaborative Research Program, SICORP for JP-US metabolomics.

\section{Author Contributions}

Nobuyuki Okahashi, Chikara Furusawa, and Hiroshi Shimizu designed research. Nobuyuki Okahashi, Shuichi Kajihata, and Chikara Furusawa performed research. Nobuyuki Okahashi and Shuichi Kajihata analyzed data. Nobuyuki Okahashi, Chikara Furusawa, and Hiroshi Shimizu wrote the manuscript.

\section{Conflicts of Interest}

The authors declare no conflict of interest.

\section{References}

1. Shirai, T.; Fujimura, K.; Furusawa, C.; Nagahisa, K.; Shioya, S.; Shimizu, H. Study on roles of anaplerotic pathways in glutamate overproduction of Corynebacterium glutamicum by metabolic flux analysis. Microb. Cell Fact. 2007, 6, 19.

2. Nielsen, J.; Jewett, M.C. Impact of systems biology on metabolic engineering of Saccharomyces cerevisiae. FEMS Yeast Res. 2008, 8, 122-131.

3. Becker, J.; Zelder, O.; Häfner, S.; Schröder, H.; Wittmann, C. From zero to hero-Design-based systems metabolic engineering of Corynebacterium glutamicum for L-lysine production. Metab. Eng. 2011, 13, 159-168. 
4. Yim, H.; Haselbeck, R.; Niu, W.; Pujol-Baxley, C.; Burgard, A.; Boldt, J.; Khandurina, J.; Trawick, J.D.; Osterhout, R.E.; Stephan, R.; et al. Metabolic engineering of Escherichia coli for direct production of 1, 4-butanediol. Nat. Chem. Biol. 2011, 7, 445-452.

5. Toya, Y.; Shimizu, H. Flux analysis and metabolomics for systematic metabolic engineering of microorganisms. Biotechnol. Adv. 2013, 31, 818-826.

6. Stephanopoulos, G. Metabolic fluxes and metabolic engineering. Metab. Eng. 1999, 1, 1-11.

7. Wiechert, W. ${ }^{13} \mathrm{C}$ metabolic flux analysis. Metab. Eng. 2001, 3, 195-206.

8. Wittmann, C. Fluxome analysis using GC-MS. Microb. Cell Fact. 2007, 6, 6.

9. Zamboni, N.; Fendt, S.-M.; Rühl, M.; Sauer, U. ${ }^{13}$ C-based metabolic flux analysis. Nat. Protoc. 2009, 4, 878-892.

10. Christensen, B.; Nielsen, J. Isotopomer analysis using GC-MS. Metab. Eng. 1999, 1, 282-290.

11. Szyperski, T. Biosynthetically directed fractional ${ }^{13} \mathrm{C}$-labeling of proteinogenic amino acids. An efficient analytical tool to investigate intermediary metabolism. Eur. J. Biochem. 1995, 232, 433-448.

12. Krömer, J.O.; Sorgenfrei, O.; Klopprogge, K.; Heinzle, E.; Wittmann, C. In-depth profiling of lysine-producing Corynebacterium glutamicum by combined analysis of the transcriptome, metabolome, and fluxome. J. Bacteriol. 2004, 186, 1769-1784.

13. Rühl, M.; Coq, D.L.; Aymerich, S.; Sauer, U. ${ }^{13}$ C-flux analysis reveals NADPH-balancing transhydrogenation cycles in stationary phase of nitrogen-starving Bacillus subtilis. J. Biol. Chem. 2012, 287, 27959-27970.

14. Iwatani, S.; Dien, S.V.; Shimbo, K.; Kubota, K.; Kageyama, N.; Iwahata, D.; Miyano, H.; Hirayama, K.; Usuda, Y.; Shimizu, K.; et al. Determination of metabolic flux changes during fed-batch cultivation from measurements of intracellular amino acids by LC-MS/MS. J. Biotechnol. 2007, 128, 93-111.

15. Rühl, M.; Zamboni, N.; Sauer, U. Dynamic flux responses in riboflavin overproducing Bacillus subtilis to increasing glucose limitation in fed-batch culture. Biotechnol. Bioeng. 2010, 105, 795-804.

16. Wittmann, C.; Hans, M.; Heinzle, E. In vivo analysis of intracellular amino acid labelings by GC/MS. Anal. Biochem. 2002, 307, 379-382.

17. Mori, E.; Furusawa, C.; Kajihata, S.; Shirai, T.; Shimizu, H. Evaluating ${ }^{13} \mathrm{C}$ enrichment data of free amino acids for precise metabolic flux analysis. Biotechnol. J. 2011, 6, 1377-1387.

18. Nöh, K.; Wiechert, W. The benefits of being transient: Isotope-based metabolic flux analysis at the short time scale. Appl. Microbiol. Biotechnol. 2011, 91, 1247-1265.

19. Toya, Y.; Ishii, N.; Nakahigashi, K.; Hirasawa, T.; Soga, T.; Tomita, M.; Shimizu, K. ${ }^{13}$ C-metabolic flux analysis for batch culture of Escherichia coli and its pyk and pgi gene knockout mutants based on mass isotopomer distribution of intracellular metabolites. Biotechnol. Prog. 2010, 26, 975-992.

20. Ishii, N.; Nakahigashi, K.; Baba, T.; Robert, M.; Soga, T.; Kanai, A.; Hirasawa, T.; Naba, M.; Hirai, K.; Hoque, A.; et al. Multiple high-throughput analyses monitor the response of E. coli to perturbations. Science 2007, 316, 593-597.

21. Fischer, E.; Sauer, U. Metabolic flux profiling of Escherichia coli mutants in central carbon metabolism using GC-MS. Eur. J. Biochem. 2003, 270, 880-891. 
22. Antoniewicz, M.R.; Kraynie, D.F.; Laffend, L.A.; Gonzales-Lergier, J.; Kelleher, J.K.; Stephanopoulos, G. Metabolic flux analysis in a nonstationary system: Fed-batch fermentation of a high yielding strain of E. coli producing 1,3-propanediol. Metab. Eng. 2007, 9, 277-292.

23. Wittmann, C.; Krömer, J.O.; Kiefer, P.; Binz, T.; Heinzle, E. Impact of the cold shock phenomenon on quantification of intracellular metabolites in bacteria. Anal. Biochem. 2004, 327, 135-139.

24. Bolten, C.J.; Kiefer, P.; Letisse, F.; Portais, J.-C.; Wittmann, C. Sampling for metabolome analysis of microorganisms. Anal. Chem. 2007, 79, 3843-3849.

25. Ingraham, J.L.; Maaløe, O.; Neidhardt, F.C. Growth of the Bacterial Cell; Sinauer Associates Inc.: Sunderland, MA, USA, 1983; p. 128.

26. Van Winden, W.A.; Wittmann, C.; Heinzle, E.; Heijnen, J.J. Correcting mass isotopomer distributions for naturally occurring isotopes. Biotechnol. Bioeng. 2002, 80, 477-479.

27. Antoniewicz, M.R.; Kelleher, J.K.; Stephanopoulos, G. Accurate assessment of amino acid mass isotopomer distributions for metabolic flux analysis. Anal. Chem. 2007, 79, 7554-7559.

28. Kajihata, S.; Furusawa, C.; Matsuda, F.; Shimizu, H. OpenMebius: An open source software for isotopically nonstationary ${ }^{13} \mathrm{C}$-based metabolic flux analysis. BioMed Res. Int. 2014, in press.

29. Antoniewicz, M.R.; Kelleher, J.K.; Stephanopoulos, G. Elementary metabolite units (EMU): A novel framework for modeling isotopic distributions. Metab. Eng. 2007, 9, 68-86.

30. Kleijn, R.J.; van Winden, W.A.; Ras, C.; van Gulic, W.M.; Schipper, D.; Heijnen, J.J. ${ }^{13}$ C-labeled gluconate tracing as a direct and accurate method for determining the pentose phosphate pathway split ratio in Penicillium chrysogenum. Appl. Environ. Microbiol. 2006, 72, 4743-4754.

31. Costenoble, R.; Müller, D.; Barl, T.; van Gulic, W.M.; van Winden, W.A.; Reuss, M.; Heijnen, J.J. ${ }^{13} \mathrm{C}$-Labeled metabolic flux analysis of a fed-batch culture of elutriated Saccharomyces cerevisiae. FEMS Yeast Res. 2007, 7, 511-526.

32. Antoniewicz, M.R.; Kelleher, J.K.; Stephanopoulos, G. Determination of confidence intervals of metabolic fluxes estimated from stable isotope measurements. Metab. Eng. 2006, 8, 324-337.

(C) 2014 by the authors; licensee MDPI, Basel, Switzerland. This article is an open access article distributed under the terms and conditions of the Creative Commons Attribution license (http://creativecommons.org/licenses/by/3.0/). 